\title{
Evolution of the coefficient of lateral earth pressure at rest with interparticle friction: a numerical study
}

\author{
Hoang $C$ Nguyen $^{1 *}$ \\ ${ }^{1}$ Former PhD student, Department of Civil and Environmental Engineering, Imperial College London, London, UK
}

\begin{abstract}
The grain-scale nature of evolution of the coefficient of lateral earth pressure at rest $\left(K_{0}\right)$ with interparticle friction $\left(\mu_{p}\right)$ is poorly understood. This study aims to use discrete element method simulations of vertical one-dimensional compression on both face centred cubic (FCC) samples and random monodisperse (RM) samples to link $K_{0}$ and $\mu_{p}$, and the results show that $K_{0}$ increases with reductions in interparticle friction. Although $K_{0}$ is dependent upon the sample density, patterns of evolutions with strain levels are likely to be unchanged with initial confining pressures. The stress-induced fabric becomes more anisotropic for samples with high values of the interparticle friction. The percentage of particles with a high value of the normal contact force increases with increasing strain levels as the interparticle friction increases in the simulations.
\end{abstract}

\section{Introduction}

Particulate discrete element modelling (DEM) is now well established and provides a wonderful research tool for many branches of engineering science in general, and in geomechanics in particular. This method was proposed in the late 1970s [1] and the steady increase in the number of DEM-related publications published each year show the potential of this numerical method [2]. For geomechanics studies, particulate DEM is relatively simple in terms of input parameters but effective in capturing the key elements of soil behaviour. Numerical DEM analyses allow geomechanics researchers to understand what happens at the particle scale which provides a useful explanation of the overall responses. As outlined in [2], two main motivations for DEM models lie in: (i) link between microscale interactions and macro-scale response; and (ii) analysis of large displacement problems in geomechanics. Use of a modified version of the open-source code, Granular LAMMPS [3] allows the highly complex behaviour of granular materials to be successfully captured. Not only can the boundary condition be easily mimicked the conditions that earth pressure is at rest, but micro-scale data gives more insights into grain-scale mechanisms underlying the evolution of $K_{0}$ with the conditions that allow particle slide each other at contacts (i.e. $\mu_{p}$ ). Several 1D compression tests were performed, allow the link between $K_{0}$ and $\mu_{p}$ to be explored. It is usual practice to relate $K_{0}$ to the effective friction angle of soil, $\phi^{\prime}$ as $K_{0}=1-\sin \phi^{\prime}$ [4]. Herein the ratio of the ratio of horizontal effective stress, $\sigma_{h}^{\prime}$ to vertical effective stress, $\sigma_{v}^{\prime}$ is used to quantify evolution of $K_{0}$ and strain levels due to varying $\mu_{p}$.

*e-mail: h.nguyen15@imperial.ac.uk

A video is available at https://doi.org/10.48448/ef8f-7540

\section{DEM simulations}

Simulations of 1D compression tests were performed on both the FCC samples and the RM samples using a modified version of the granular LAMMPS code, with a simplified Hertz-Mindlin contact model. The FCC packing consisting of 3,200 spherical particles (the particle diameter of $2.54 \mathrm{~mm}$ ) that correspond to samples previously used in [5-7] was adopted, while the RM sample was created from an initial cloud of 34, 986 non-contacting monodisperse spherical particles (i.e. the particle diameters ranged from 2.3 to $2.7 \mathrm{~mm}$ ). Specifically, the input parameters used were shear modulus $G_{p}=25 \times 10^{9} \mathrm{~Pa}$, particle Poisson's ratio, $\mu_{p}=0.2$, particle density, $\rho_{p}=2230 \mathrm{~kg} / \mathrm{m}^{3}$ and local damping coefficient, $c_{p}=0.01$. The samples were enclosed in the vertical direction by two rigid, planar, horizontal boundaries; periodic boundaries were used in the lateral direction. Herein four values of interparticle frictions which are $\mu_{p}=0.05 ; 0.25 ; 0.50$ and 0.75 were used to assess the changes in $K_{0}$ with strain levels as $\mu_{p}$ is varied. These samples experienced two main processes. In the first step, all samples were isotropically compressed to three different values of confining pressure (i.e. $p_{0}=100 ; 300$ and $1000 \mathrm{kPa}$ ); the void ratio of samples at this stage is denoted as $e_{0}$, which is dependently upon the value of $\mu_{p}$ for the RM samples. The second step relates to $1 \mathrm{D}$ compressions in which the lateral boundary was fixed, while the top rigid wall was moved at a constant velocity $\dot{\epsilon}_{z}=0.1 \mathrm{~m} / \mathrm{s}$.

\section{DEM results}

\subsection{Macro response}

Macro-scale responses under 1D compression tests were shown in Figure 1 for the FCC packings where the deviator stress increases as $\mu_{p}$ increases. However, the mean 
effective stress, $p_{0}^{\prime}$ is likely to be independent upon on $\mu_{p}$, most probably due to the very stable configuration (Figure $1 \mathrm{~b}$ ); however, it is not the case for monodisperse samples where an increase in either $\mu_{p}$ or $p_{0}^{\prime}$ leads to a reduction in initial void ratio (i.e. $e_{0}$ ), agreeing well with numerical results reported in [8]. It is shown in Figure 1 that higher $K_{0}$ values were observed for the samples with smaller $\mu_{p}$. This is also a trend for the RM samples where looser samples experienced higher values of $\mu_{p}$ (Figure 2), along with an increase in the mean effective stress as $\mu_{p}$ increases.
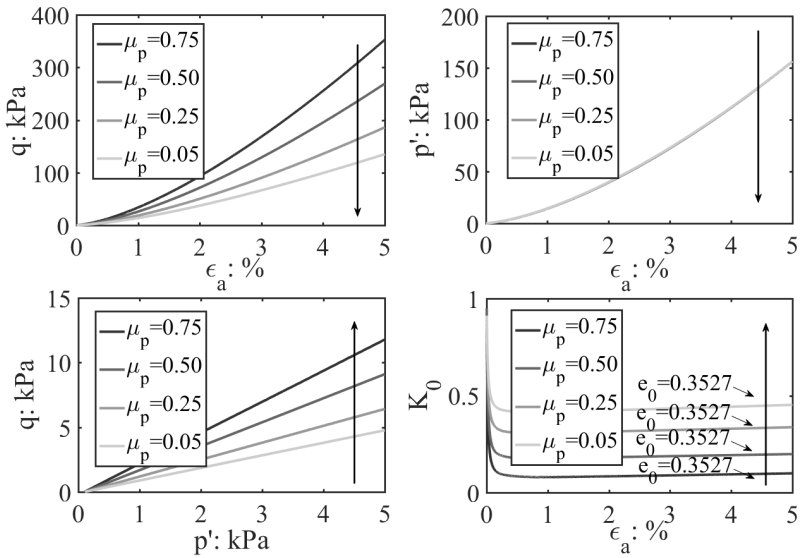

Figure 1. Dependency of macro-scale responses on interparticle friction for the FCC samples that were compressed from $p_{0}^{\prime}=$ $100 \mathrm{kPa}$
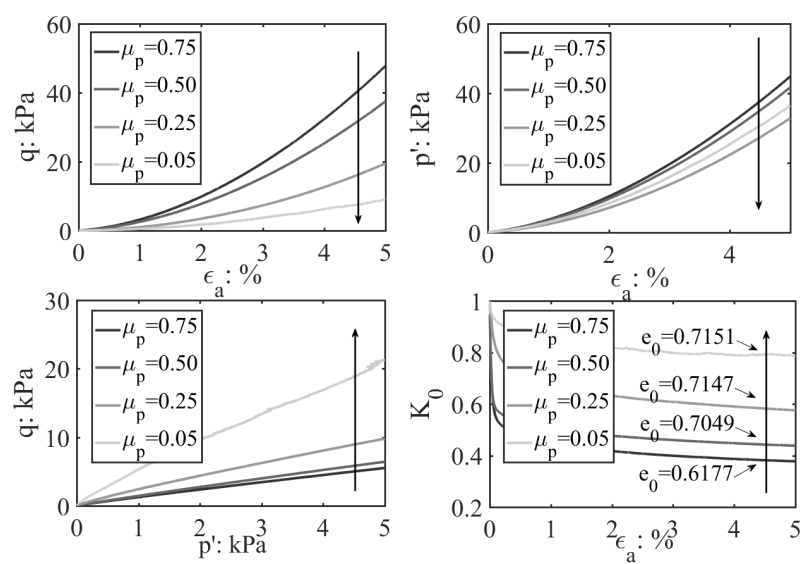

Figure 2. Dependency of macro-scale responses on interparticle friction for the RM samples that were compressed from $p_{0}^{\prime}=$ $100 \mathrm{kPa}$.

Assessments of dependency of $K_{0}$ on either the initial void ratio and mean effective confining pressure were made for both samples (Figure 3). It is observed that the $K_{0}$ values of both samples are independent upon the initial mean effective pressure. At each stress level, there is a reduction in $K_{0}$ as $\epsilon_{a}$ increases, providing a check on the numerical results obtained in [8] who performed 1D simulations on samples with particle size distributions. Interestingly, $K_{0}$ continuously reduces over a wide strain range $\left(\epsilon_{a}=0: 5 \%\right)$ for the RM samples; however, it is, in contrast to the RM samples, that $K_{0}$ is almost unchanged when $\epsilon_{a}$ is larger than $0.1 \%$ for the FCC sample.
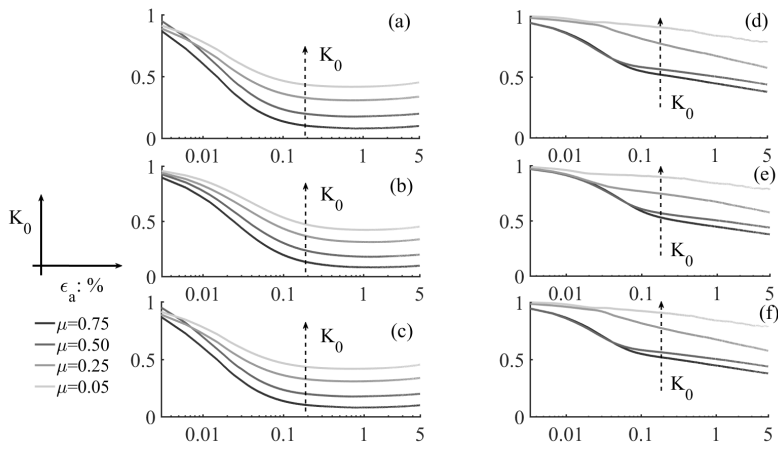

Figure 3. Dependency of macro-scale responses on interparticle friction when samples were compressed from $p_{0}^{\prime}=100 \mathrm{kPa}$ : (a) the FCC sample: $p_{0}^{\prime}=100 \mathrm{kPa}$; (b) the FCC sample: $p_{0}^{\prime}=$ $300 \mathrm{kPa}$; (c) the FCC sample: $p_{0}^{\prime}=1000 \mathrm{kPa}$; (d) the RM sample: $p_{0}^{\prime}=100 k P a$; (e) the RM sample: $p_{0}^{\prime}=300 k P a$; (f) the RM sample: $p_{0}^{\prime}=1000 \mathrm{kPa}$;

\subsection{Micro-scale analysis}

Analyses of particle-scale data extracted from DEM simulations provide additional information on macro-scale responses under one-dimensional compression. The stressinduced fabric anisotropy herein is quantified using: (i) particle orientations; (ii) contact normal orientations. Contact orientations are shown in Figure 4 and Figure 5 using rose diagrams, in which each contact is distributed on the spatial orientation. Specifically, each rose diagram has 18 bins, with angles varying from $0^{\circ}$ to $180^{\circ}$. The length of each bin represents the number of contacts, with projections onto the $\mathrm{xy}, \mathrm{xz}$ and $\mathrm{yz}$ planes. Referring to Figure 5, the stress-induced fabric is highly sensitive to the strain levels, in which the distribution of contact numbers is isotropic at $p_{0}^{\prime}=1000 \mathrm{kPa}$; however, the fabric anisotropy becomes remarkably for the verticle planes as $\epsilon_{a}$ increases to $5 \%$. In contrast, numbers of contact are more isotropic for the horizontal plane. Along with the contact distributions, evolutions of the normal force at contact were analyzed and shown in Figure 6 and Figure 7. As expected, the degree of anisotropy becomes significantly as $\mu_{p}$ increases for the vertical planes; however, in the horizontal plane, the force distributions remain in an almost isotropic manner due to its fabric isotropy.

Investigations into changes in the coordination number were analysed for both samples (Figure 8a and 8e). While the RM samples experience an increase in $N_{c}$ as $\mu_{p}$ reduces during 1D compressions, FCC packings show that $N_{c}$ tends to drop as $\mu_{p}$ increases. In addition, there is no simple link between the friction dissipation and $\mu_{p}$ for both samples (Figure $8 \mathrm{~b}$ and $8 \mathrm{f}$ ). However, the pattern of $F_{n} / F_{n}^{\max }\left(F_{n}\right.$ and $F_{n}^{\max }$ are the normal contact force and the maximum normal force at contact respectively) moves to the right as $\epsilon_{a}$ increases, indicating that more particles bear higher magnitudes of the normal force at contacts. Noting that, at $p_{0}^{\prime}=1000 \mathrm{kPa}$, the pattern of $F_{n} / F_{n}^{\max }$ is almost identical for the RM samples, regardless of the use of different values of $\mu_{p}$. Changes in the trend of $F_{n} / F_{n}^{\max }$ for the FCC samples were shown in Figure $8 \mathrm{c}$ and $8 \mathrm{c}$ where there is no simple correlation between $F_{n} / F_{n}^{\max }$ and $\mu_{p}$. 

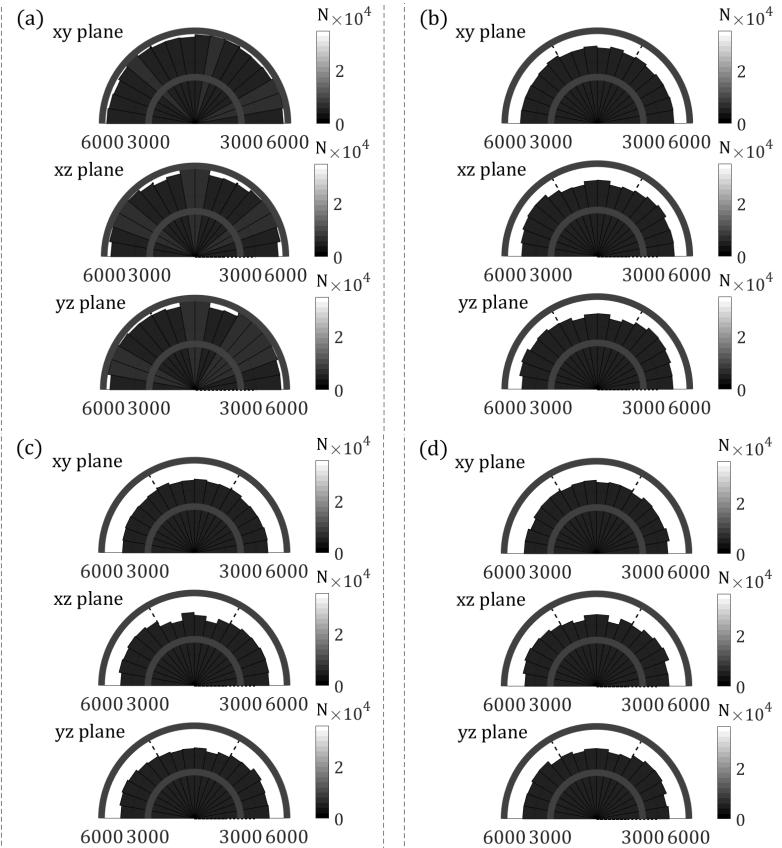

Figure 4. Contact distributions of the RM samples at $p_{0}^{\prime}=$ $1000 \mathrm{kPa}$, with projections onto the $\mathrm{xy}, \mathrm{xz}$ and $\mathrm{yz}$ planes, respectively: (a) $\mu_{p}=0.05$; (b) $\mu_{p}=0.25$; (c) $\mu_{p}=0.50$; and (d) $\mu_{p}=0.75$.
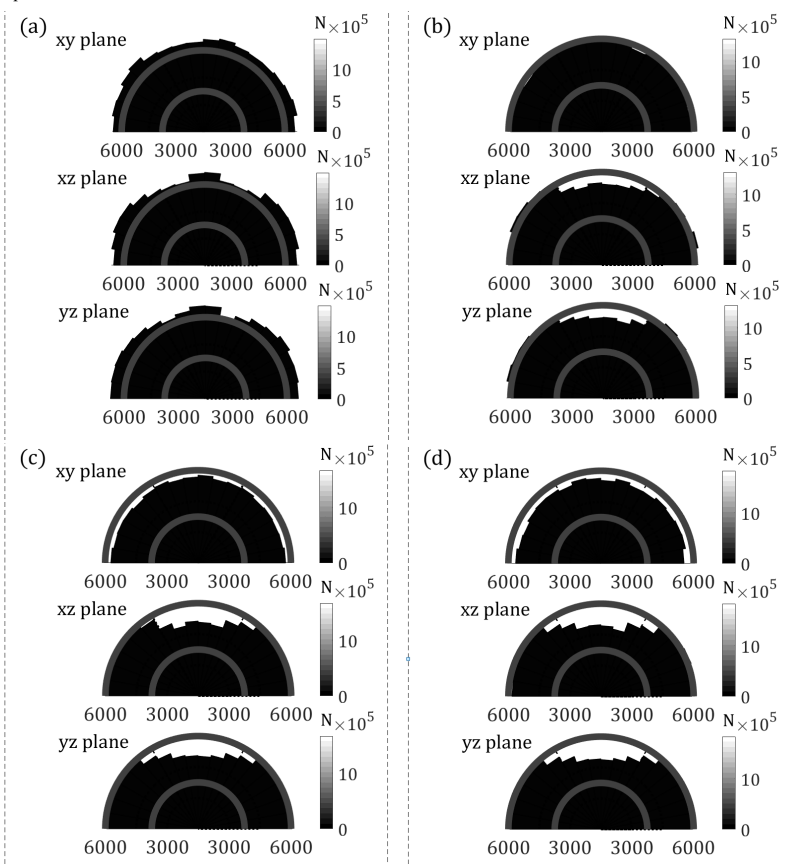
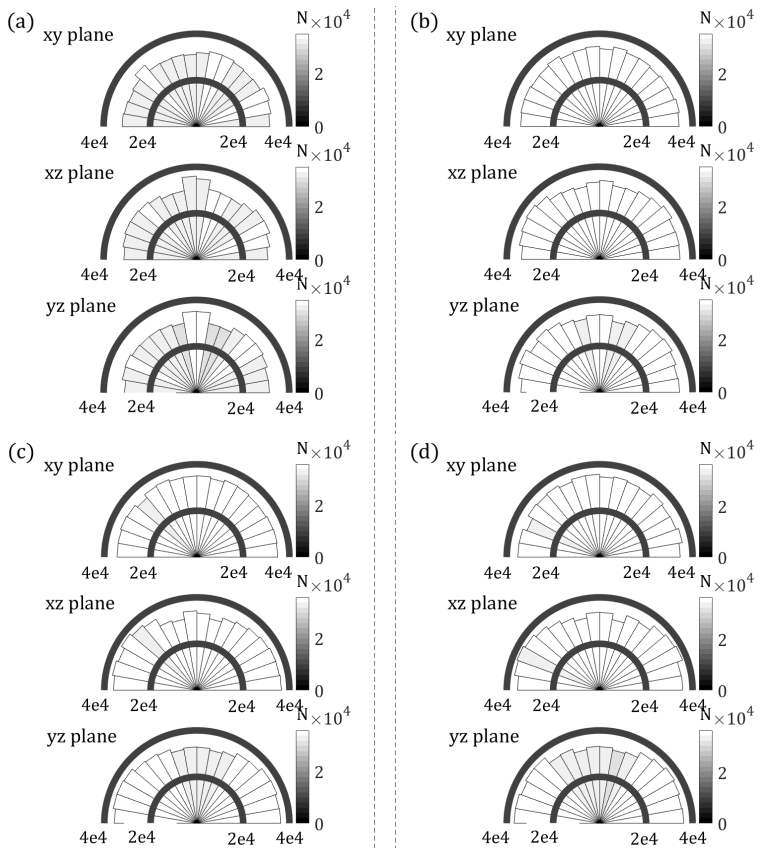

Figure 6. Normal force distributions of the RM samples at $p_{0}^{\prime}=1000 \mathrm{kPa}$, with projections onto the $\mathrm{xy}, \mathrm{xz}$ and $\mathrm{yz}$ planes, respectively: (a) $\mu_{p}=0.05$; (b) $\mu_{p}=0.25$; (c) $\mu_{p}=0.50$; and (d) $\mu_{p}=0.75$.
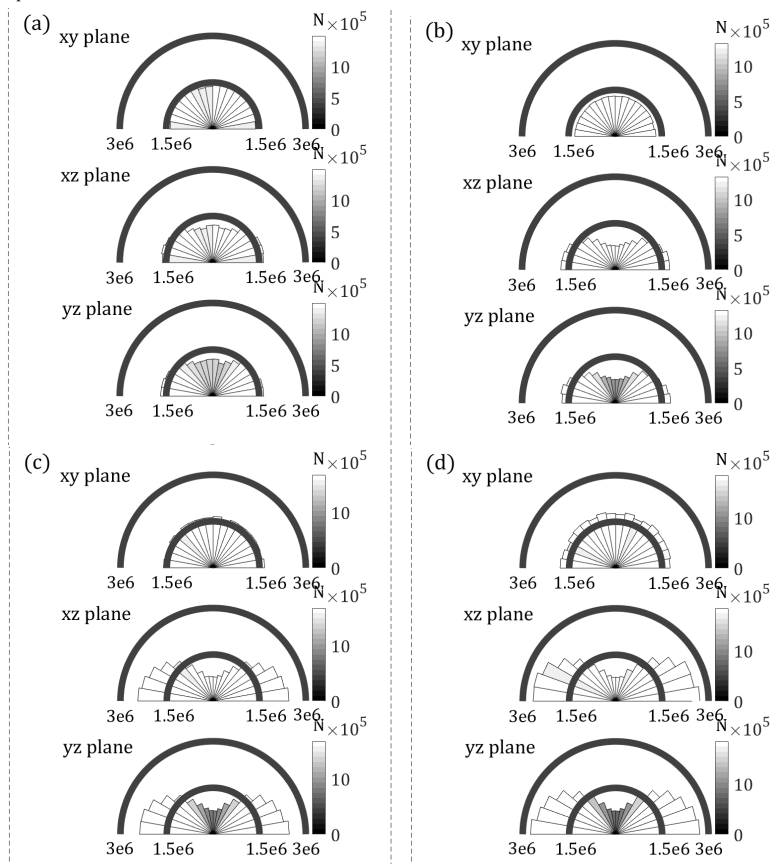

Figure 5. Contact distributions of the RM samples at $\epsilon_{a}=0.5 \%$, with projections onto the $x y, x z$ and yz planes, respectively: (a) $\mu_{p}=0.05$; (b) $\mu_{p}=0.25$; (c) $\mu_{p}=0.50$; and (d) $\mu_{p}=0.75$.

Figure $8 \mathrm{i}$ and Figure $8 \mathrm{j}$ show the normalised contact force values for the RM samples at $p_{0}=1000 \mathrm{kPa}$ and at $\epsilon_{a}=5 \%$. The value of mean normal contact force for the whole sample is denoted as $F_{n}^{\text {mean }}$ and is calculated using micro-scale data at contacts being extracted from DEM simulations. From a particle-scale perspective, the normalised contact force values represent the ability that stress is transmitted through granular materials as noted in [9]. Although the friction value has no influence on the

Figure 7. Normal force distributions of the RM samples at $\epsilon_{a}=0.5 \%$, with projections onto the xy, xz and yz planes, respectively: (a) $\mu_{p}=0.05$; (b) $\mu_{p}=0.25$; (c) $\mu_{p}=0.50$; and (d) $\mu_{p}=0.75$.

pattern of normalised contact force, there is an increase in the number of particles that bears higher normal force as the axial strain increases, with the plots of $F_{n} / F_{n}^{\text {mean }}$ being shifted to the right as shown in Figure 8j. This implies that more chains of highly stressed particles are formed during one-dimensional compression tests, where more stress is transmitted in the vertical planes than in the horizontal plane as previously shown in Figure 6 and Figure 7. 

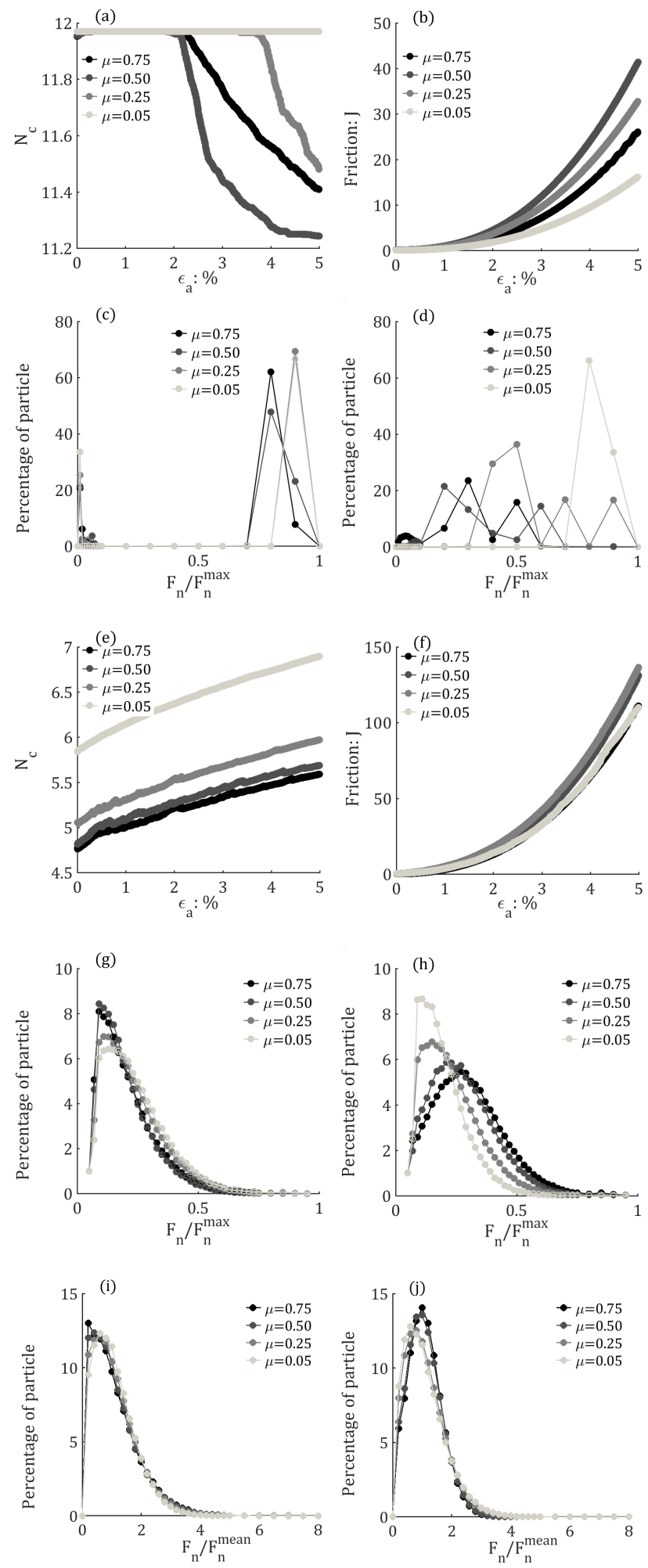

Figure 8. Evolutions of the coordination number and friction dissipation with axial strain: (a) $N_{c}$ : the FCC sample; (b) friction: the FCC sample; (c) $F_{n} / F_{n}^{\max }$ at $p_{0}^{\prime}=1000 \mathrm{kPa}$ : the FCC sample; (d) $F_{n} / F_{n}^{\max }$ at $\epsilon_{a}=5 \%$ : the FCC sample; (e) $N_{c}$ : the RM sample; (f) friction: the RM sample; (g) $F_{n} / F_{n}^{\max }$ at $p_{0}^{\prime}=1000 \mathrm{kPa}$ : the RM sample; (h) $F_{n} / F_{n}^{\max }$ at $\epsilon_{a}=5 \%$ : the RM sample; (i) $F_{n} / F_{n}^{\text {mean }}$ at $p_{0}^{\prime}=1000 \mathrm{kPa}$ : the RM sample; and (j) $F_{n} / F_{n}^{\text {mean }}$ at $\epsilon_{a}=5 \%:$ the RM sample.

\section{Conclusions}

The link between $K_{0}$ and $\mu_{p}$ has been described using onedimensional DEM simulations, arriving at the observation that $K_{0}$ decreases as $\mu_{p}$ increases. Although $K_{0}$ depends on the sample density and the material fabric, there is no dependency of $K_{0}$ on the confining pressure. While the FCC sample experiences a drop in the coordination number as the strain level increases, it is observed that there is a rise in the coordination number for the RM samples. There is no simple link between the friction dissipation and the interparticle friction; however, the coordination number increases with increasing interparticle friction for the randomly monodisperse size sample. The pattern of $F_{n} / F_{n}^{\text {max }}$ moves to the right as the strain level increases, indicating that more particles bear the high magnitude of the normal contact force. The extent of the strain-induced anisotropy depends on the $K_{0}$ value, with higher degree of anisotropy for samples with larger values of the interparticle friction. The results of this study support the prior findings reported in [8] who confirmed that the values of $K_{0}$ depend upon the internal structure which is caused herein by using different values of $\mu_{p}$ during compression tests.

\section{References}

[1] P.A. Cundall, O.D.L. Strack, Geotechnique 29, 19 (1979)

[2] C. O'Sullivan, Particulate discrete element modelling: a geomechanics perspective (Taylor \& Francis, 2011)

[3] S. Plimpton, J. Comput. Phys. 117, 20 (1995)

[4] J. Jaky, J. Soc. Hung. Eng. Arch 78, 4 (1944)

[5] H.C. Nguyen, Micromechanics of shear wave propagation and non-linear stiffness of granular materials, $\mathrm{PhD}$ thesis, Imperial College London, (2020)

[6] H.C. Nguyen, M. Otsubo, C. O'Sullivan, EPJ Web of Conferences 140, 4 (2017)

[7] H.C. Nguyen, C. O'Sullivan, M. Otsubo, Geotech. Lett. 8, 7 (2018)

[8] J.C. Lopera Perez, C.Y. Kwok, C. O’Sullivan, X. Huang, K.J. Hanley, Geotech. Lett. 5, 8 (2015)

[9] F.Radjai, M. Jean, J. J. Moreau, and S. Roux, Phys. Rev. Lett. 77, 4 (1996) 